\title{
Negative impedance interval of blood flow in capillary bed
}

\author{
Gyula Vincze ${ }^{1}$, Gyula Peter Szigeti ${ }^{2}$, Oliver Szasz ${ }^{1}$ \\ ${ }^{1}$ St. Istvan University, Department of Biotechnics, Hungary \\ biotech@gek.szie.hu
}

${ }^{2}$ Institute of Human Physiology and Clinical Experimental Research, Semmelweis University, Hungary

\section{ABSTRACT}

We study a simple model of the capillary network of living systems. The volume flow of the blood versus pressure drop has a negative impedance interval, because the viscosity of the blood decreases with increasing shear rate. We explain the channel formation and deviations of the blood flow in solid tumours by this phenomenon.

\section{Indexing terms/Keywords}

minimal dissipation principle, capillary bed filtering, angiogenesis, negative impedance of flow

\section{INTRODUCTION}

The minimal energy-dissipation principle is valid for isothermal stationary dissipative structures. This controls the formation of the network to minimize the dissipative energy. Simple examples can be shown on direct current circuits (see Figure 1.) with $\mathrm{P}$ power I currents and $\mathrm{R}$ resistivities. In this case,

$$
P=I_{1}^{2} R_{1}+I_{2}^{2} R_{2}
$$

has to be minimal under the condition of:

$$
I=I_{1}+I_{2}
$$

Then:

and

$$
\delta P=2 I_{1} R_{1} \delta I_{1}+I_{2} R_{2} \delta I_{2}=0
$$

$$
\delta I=\delta I_{1}+\delta I_{2}=0
$$

According to the Lagrange multiplier method,

$$
\left(I_{1} R_{1}+\lambda\right) \delta I_{1}\left(+I_{2} R_{2}+\lambda\right) \delta I_{2}=0
$$

The multiplier is chosen as:

$$
\left(I_{1} R_{1}+\lambda\right)=0
$$

In this case, because the arbitrary $\delta I_{2}$ in (3) is also valid:

$$
\left(I_{2} R_{2}+\lambda\right)=0
$$

So (as is well known anyway), an equal potential will be formed on the two resistors:

$$
I_{1} R_{1}=I_{2} R_{2}
$$

Hence the current distribution from (2) is:

$$
I_{1}=I \frac{R_{2}}{R_{1}+R_{2}}
$$

The perturbed currents are denoted by $I_{1}^{*}, I_{2}^{*}$, and the perturbed condition according to (2) is

$$
\begin{aligned}
& \delta P=\left(I_{1}^{*}+\delta I_{1}\right)^{2} R_{1}+\left(I_{2}{ }^{*}+\delta I_{2}\right)^{2} R_{2}-\left(I_{1}{ }^{*}\right)^{2} R_{1}+\left(I_{2}{ }^{*}\right)^{2} R_{2}= \\
& =\left(2 I_{1}{ }^{*} R_{1} \delta I_{1}+2 I_{2}{ }^{*} R_{2} \delta I_{2}\right)+\left(\delta I_{1}^{2} R_{1}+\delta I_{2}^{2} R_{2}\right)=\delta I_{1}^{2} R_{1}+\delta I_{2}^{2} R_{2} \geq 0
\end{aligned}
$$


where we used $\delta I_{1}=-\delta I_{2}$ from (8). The perturbed state has higher dissipation than the regularly calculated standard circuit, so the regular circuits has minimal energy dissipation.

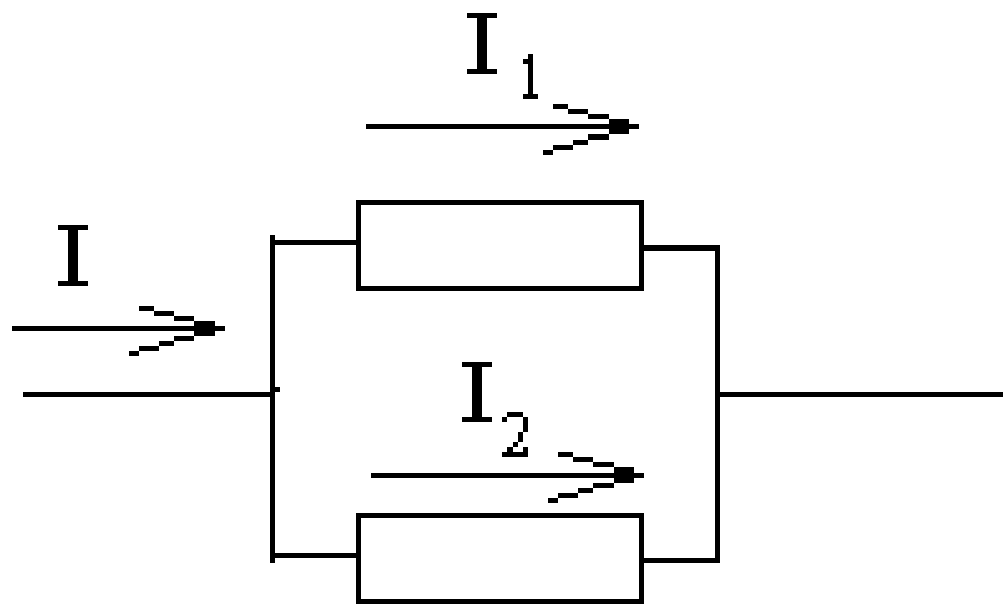

Figure 1. Demonstration of the minimal energy dissipation principle. This circuit divides the actual current, keeping the dissipation to a minimum.

\section{METHOD}

A simple model of the velocity profile of the capillary is shown in Figure 2. [1], [2].

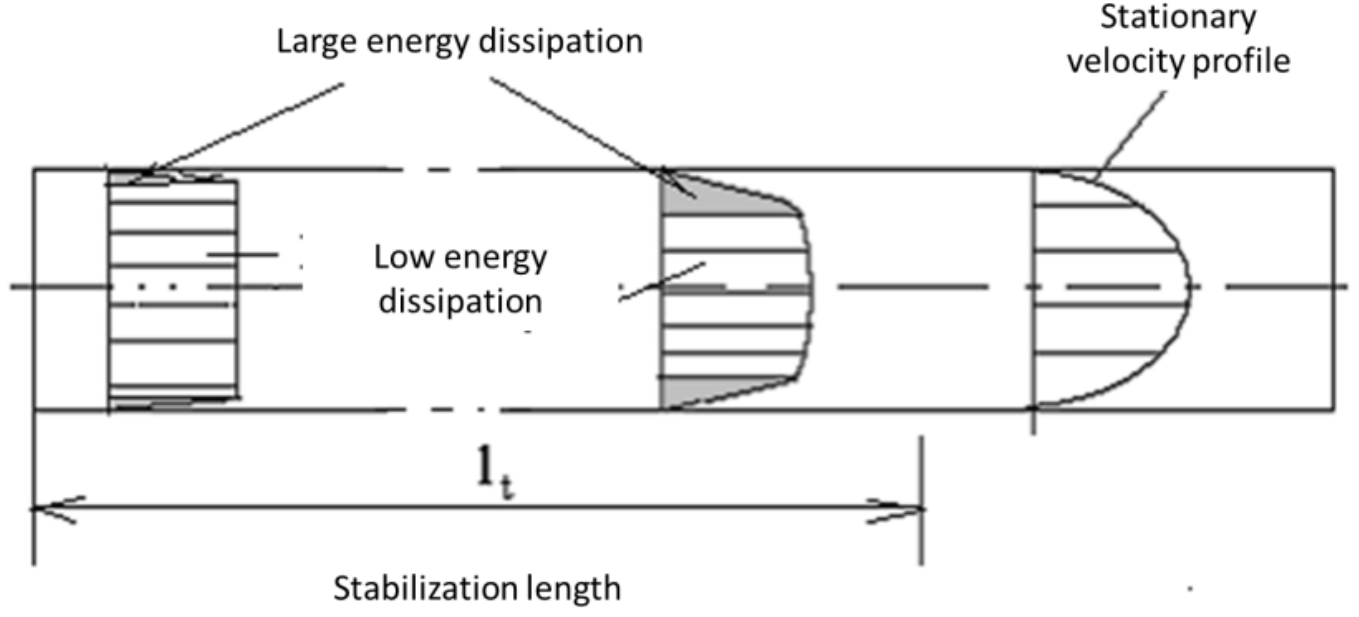

Figure 2. Formation of stationary state at the entrance of the actual vessel section. There is a drop of pressure of the flow, and the velocity profile forms step by step.

The transition interval also has a pressure drop due to the energy dissipation there.

We hypothesize that the pressure drop at the incoming interval is smaller in the case of blood than the other stationary velocity profile belonging to the same size of transition interval. This hypothesis is supported by the high shear rate (the change of the velocity in the unit radius) and the viscosity of the blood decreases with increasing shear rate (see Figure 3.), [3]. The energy dissipation in the unit volume is:

$$
p=\frac{\eta}{2} D^{2}
$$

So the situation proposed by the hypothesis could happen. 


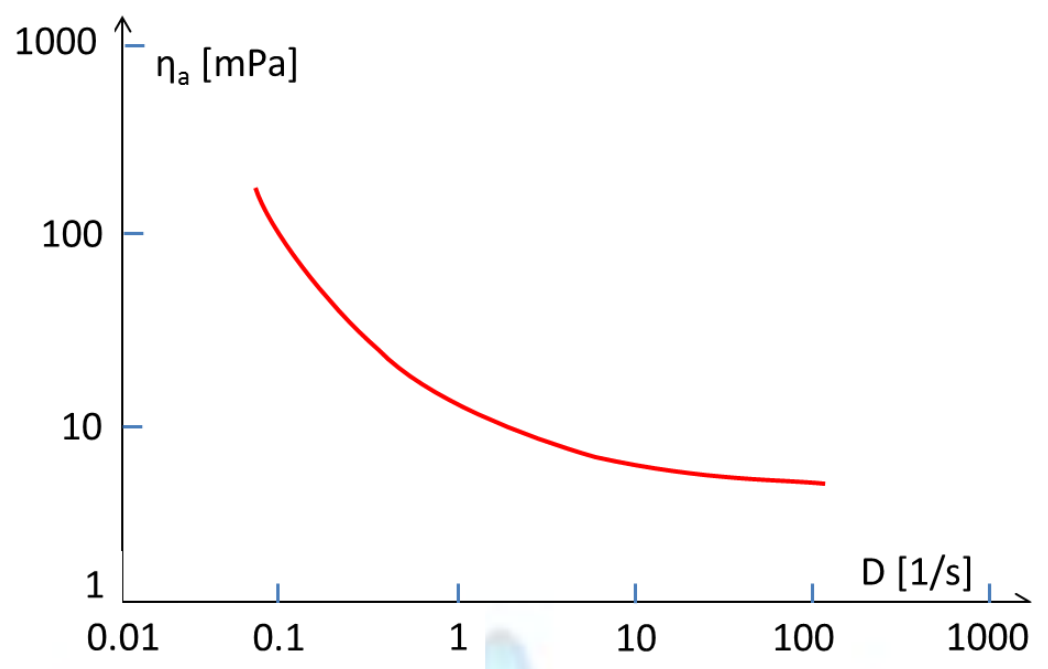

Figure 3. Apparent viscosity of blood versus shear rate

Note that the opposite situation occurs in the case of normal non-Newtonian fluids, but the deviation is smaller than in the case of Newtonian fluids, because the viscosity of the latter is constant. However in the case of power-type nonNewtonian fluids:

$$
\eta=\frac{\tau}{D}=\frac{K D^{m}}{D}=K D^{m-1}
$$

where $m-1<0$.

According to the experiments

$$
l_{t}=c d \operatorname{Re}
$$

where $d$ is the vessel's diameter and $c \approx 0.05$. In the case of an increasing volume flow, the pressure drop decreases because the transition interval increases. The decrease of the pressure reverses due to the formation of turbulence, causing an increasing pressure versus volume flow, see Figure 4.

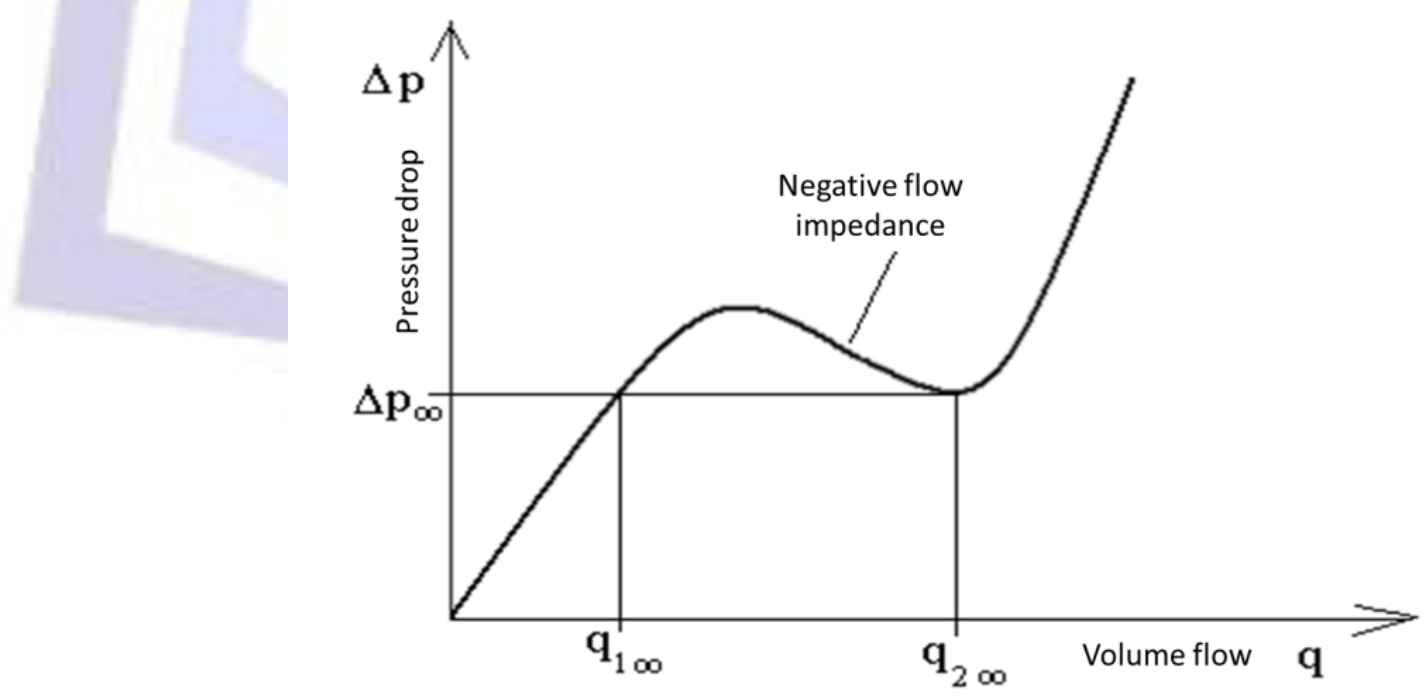

Figure 4. Hypothesized characteristic of the capillary set in the network

\section{RESULTS}

The capillary beds work like a filter (see Figure 5.) [4], [5]. When the characteristic is negative, as in Figure 4., a current channel forms, the homogeneous current distribution is broken, and one part of the filter will have a higher volume flow than the other. 


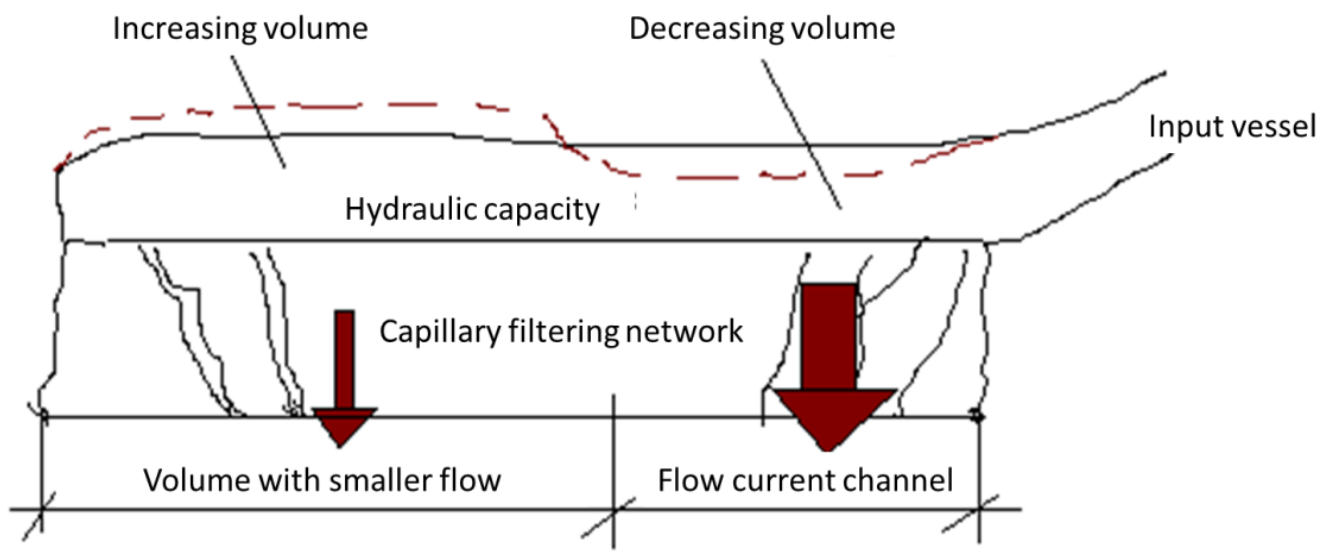

Figure 5. The capillary set works like a filter in the flow

The energy dissipation of the filter induces this effect:

$$
P=\Delta p_{1} q_{1}+\Delta p_{2} q_{2}
$$

where the subscript 1 denotes the smaller and larger volume currents. The pressure is unique:

$$
\Delta p_{1}=\Delta p_{2}=\Delta p
$$

Hence

$$
P=\Delta p\left(q_{1}+q_{2}\right)=\Delta p q
$$

where $q=q_{1}+q_{2}$ are the actual volume currents. Under this condition, the energy dissipation will be minimal when:

$$
P=\Delta p_{1} q_{1}+\Delta p_{2} q_{2}
$$

and $\Delta p$ is minimal. This point has pressure $\Delta p_{\infty}$ and volume-current $q_{2 \infty}$ according to Fig. 4 . On the other side of the filter the volume-current is $q_{1 \infty}$, so the current channel exists. The balance of the volume-current is

$$
\frac{a}{A} q_{2 \infty}+\frac{A-a}{A} q_{1 \infty}=q
$$

Hence,the ratio of the surfaces is:

$$
\frac{a}{A}=\frac{q-q_{1 \infty}}{q_{2 \infty}-q_{1 \infty}}
$$

There is a criterion for forming the channel. When the volume-current is unified in the area of the filter, it is perturbed with a value:

$$
\frac{a}{A}\left(q^{*}-q\right)=\delta q
$$

When q volume-current flows through, causes differential hydraulic resistance $\Delta R_{\text {diff }}:=\left(\frac{d \Delta p}{d q}\right)_{q}$, then the pressure perturbation will be:

$$
\delta p=\Delta R_{\text {diff }} \delta q
$$

The perturbation of volume-current will be absorbed by the massive supply of the incoming vessels or may allow volume changes like hydraulic condensers do (Fig. 5). When their capacity is $C_{h c}$ then 


$$
C_{h c} \frac{d \delta p}{d t}=-\delta q
$$

Consequently from (21) and (22):

$$
\frac{d t \delta q}{d t}=-\left(C \Delta R_{\text {diff }}\right)^{-1} \delta q
$$

Hence, when

$$
\Delta R_{\text {diff }}<0
$$

then the perturbation grows exponentially.

\section{DISCUSSION}

The following consequences are possible:

- In bypass operations, When vessel is taken from other part of the body, expecting independent vessels to share the job, two blood circuits are constructed because the shunt vessels between main flows does not exist ever more. Then the volume-current may flow asymmetrically and the working-point in resistance of characteristics (Fig.6. ) may be on the negative slope. Consequently asymmetric blood supply could happen, which could be measured even by temperature distribution too.

- The cancerous tumour organizes its blood supply by angiogenesis. When any instability occurs in the development, the channels discussed above could form easily.

- The blood pressure drop versus volume-current depends on the temperature and differs in the tumour from its healthy counterpart (see Figure 7.), [6]. This is because the volume-current flow starts to grow by vasodilatation. The system could work in a negative work-point in lower pressure than in the normothermia situation.
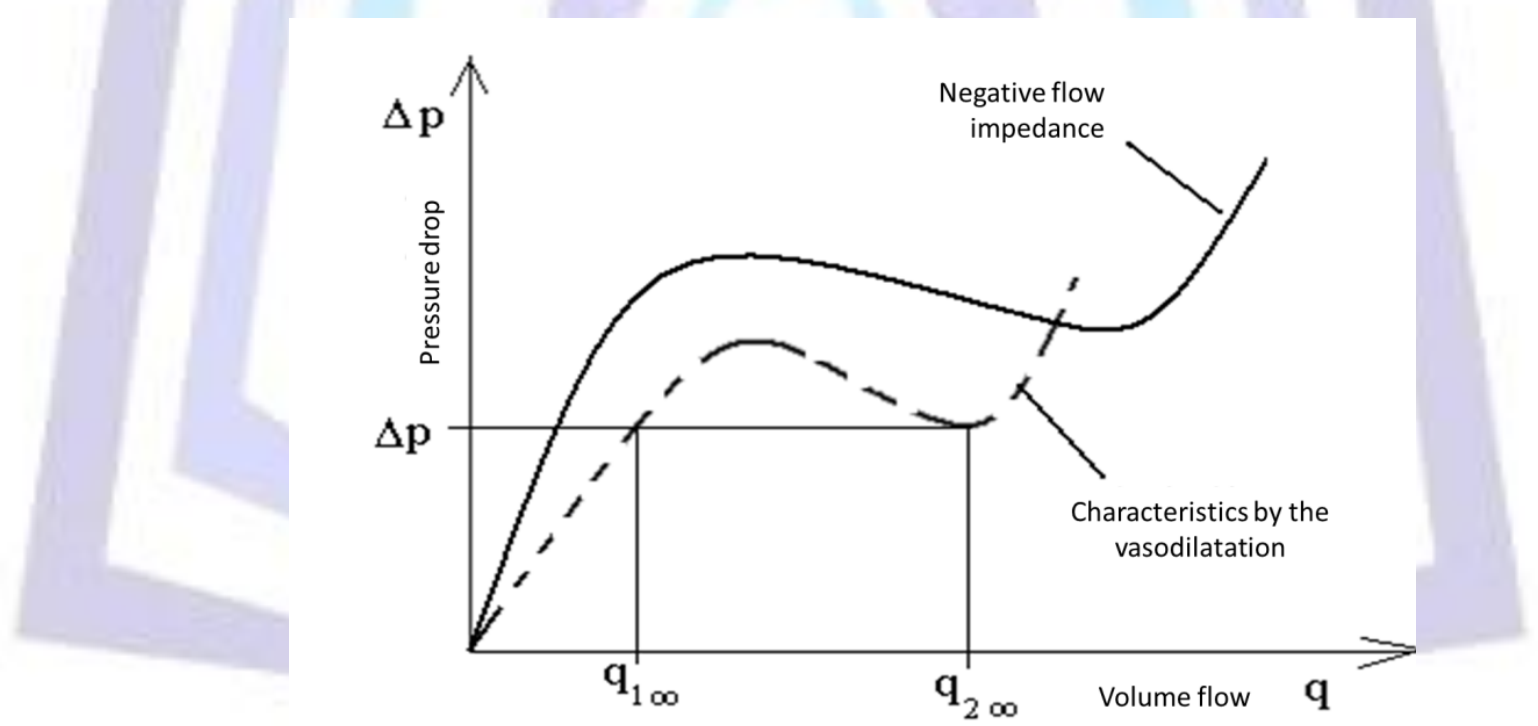

Figure 6. Change of the hydraulic characteristics of a capillary set (filter) with changes of vasodilatation by temperature increase

\section{CONCLUSION}

The supposed negative flow impedance of blood due to the characteristics of pressure drop versus volume flow could explain some particularities of the angiogenesis of solid tumours and could also be a useful tool to approximate the consequences of vasodilatation or vasoconstriction caused by various conditional changes in the tumour environment, including hyperthermia.

\section{REFERENCES}

[1] Böhme, G. 1981. Strömungsmechanik nicht-newtonsche Fluide. Teubner-Verlag, Stuttgart. 
[2] Bishop, J. J. 2001. Rheology effects of red blood cell aggregation int he venous network: A review of recent studies. Biorheology. 38:263-274.

[3] Krell, U. S., Schirser, W. 1987. Nicht-Newtonsche Flüssigkeiten. VEB Deutscher Verlag für Grundstaffindustrie, Leipzig.

[4] Taylor, A. E. 1981. Capillary fluid filtration, Starling forces and lymph flow. Circulation Research. 49(3):557-575.

[5] Yuan, S.Y., Rigor, R. R. 2010. Regulation of Endothelial Barrier Function. San Rafael (CA): Morgan and Claypool Life Sciences, Ch. 2. Structure and Function of exchange microvessels.

[6] Szasz, A., Szasz, N., Szasz, O. 2010. Oncothermia - Principles and practices. Springer Science, Heidelberg.

\section{Author' biography with Photo}

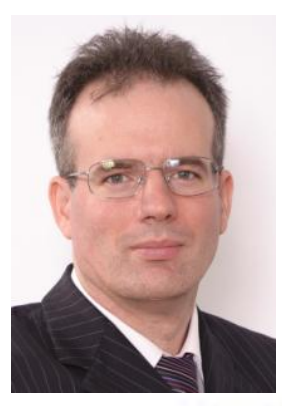

PD Dr. Oliver SZASZ, PhD

Dr. Oliver Szasz is bioengineer, associate professor of Biotechnics Department of St. Istvan University (Hungary). He has over 40 published articles, he owns several patents. He has essential contribution of inventing of nanothermia technology.

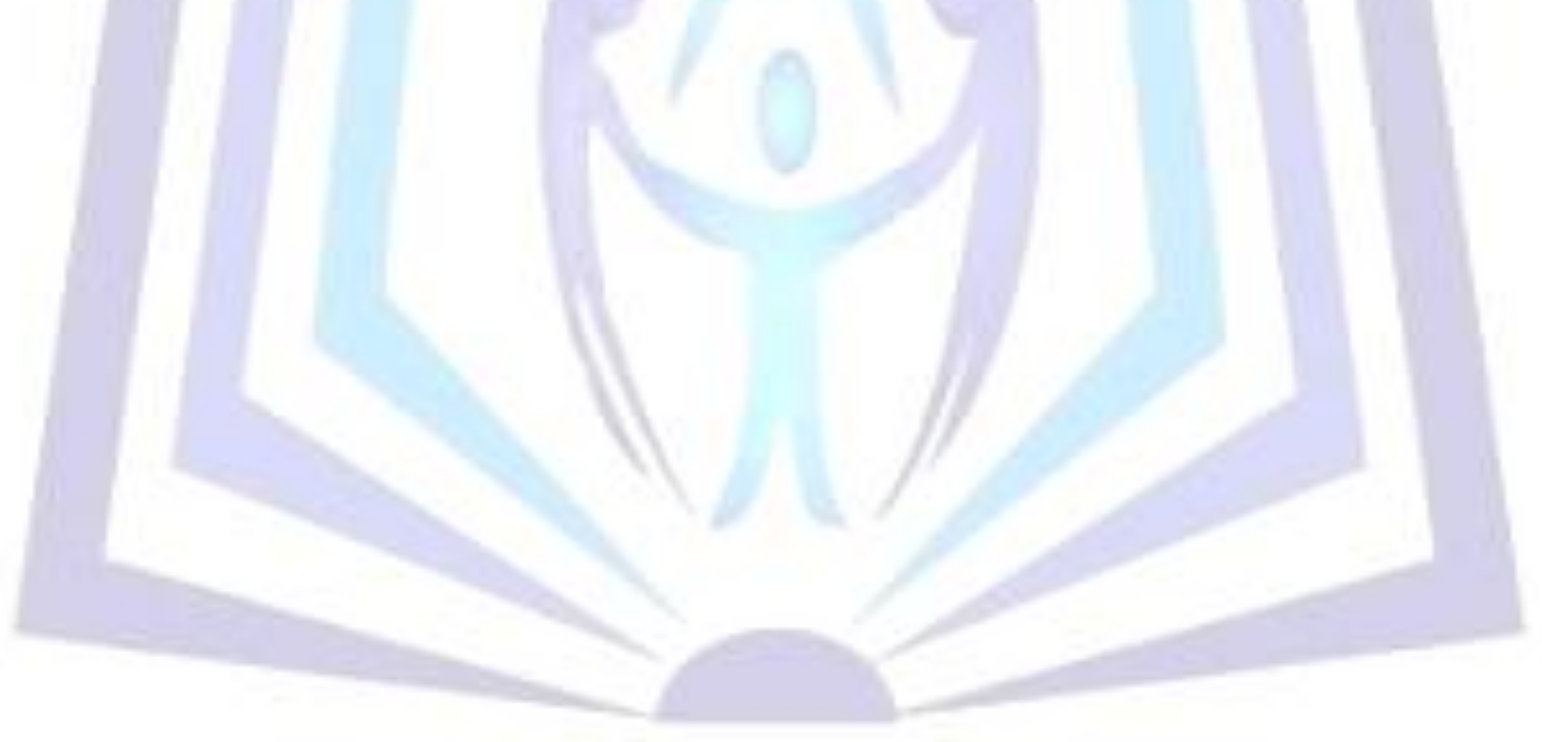

